\title{
Place recognition and way finding by children and adults
}

\author{
EDWARD H. CORNELL, C. DONALD HETH, and DENISE M. ALBERTS \\ University of Alberta, Edmonton, Alberta, Canada
}

\begin{abstract}
Children and adults were escorted on their first walk across our university campus and were periodically led off the original route during the return trip. During the return, we stopped prior to intersections on and off the original route to obtain estimates of place recognition accuracy and confidence. The subjects were then asked to point to the path that led back to the start and were corrected if wrong. Accuracy of place recognition was intermediate in a way-finding task requiring reversal of an incidentally learned novel route. However, accuracy increased as subjects were farther from the original route, indicating that the presence of novel landmarks boosted the discrimination of old and new places. Eight-year-old children were less accurate than 12-year-old children and 25-year-old adults, who did not differ in accuracy. There was a similar age difference in the ability to point to the direction to return when subjects correctly recognized that they were off route. The results are used to develop a model of way finding by place recognition.
\end{abstract}

If we are at a place where we have been before and we correctly identify it as such, we are showing place recognition in situ. The archives of experimental psychology do not include much study of this phenomenon, even though it has everyday significance. Here, we examine place recognition in the context of human way finding. We are especially interested in how recognition processes allow us to return along a route that we have taken through novel territory.

There are reasons of ecology, theory, and measurement for assessing place recognition in the context of a route reversal task. Ecologically, it can be noted that the requirements of route reversal are common; travelers often return to the origin of their excursion by the same paths that they have used to reach a destination. During route reversal, travelers have the advantage of familiarity of landmarks along the route to prevent inefficient wandering, even though they view these landmarks from a different perspective than when they first encountered them.

Navigation based on recognition of landmarks seems routine, yet most of us have experienced difficulties in situations that require that we find our way back along a route when we have not anticipated having to do so. In these situations, landmarks may be familiar, but their relationships to choice points along the route may not have been noted. Route reversal following such incidental learning may reveal important limitations of way-finding processes. For example, case histories of lost children in-

This research was supported by grants to E.H.C. from the Natural Sciences and Engineering Research Council of Canada. We thank Maurissa Abecassis, Alinda Friedman, and David Hall for help with the manuscript. Correspondence concerning this article should be addressed to E. H. Cornell, Department of Psychology, University of Alberta, Edmonton, AB, Canada T6G 2E1 (e-mail: ecornell@psych. ualberta.ca). dicate instances in which children have accompanied knowledgeable individuals to new sites but inadvertently become separated from the original party (Syrotuck, 1979).

Theoretically, the requirement to remember events that are spatially and serially reordered can be used as a particularly revealing test of representational abilities (Piaget, 1969). For example, Brown (1976) assessed the route reversal abilities of children who were classified as preoperational with reference to Piagetian logical operations such as reversibility. The children were asked to reconstruct a circuitous route taken by a toy elephant through several locations in an environmental model. Preoperational children showed no difference in forward and backward route reconstruction when the locations visited by the elephant were marked. These results suggest that one of the major difficulties in route reversal consists in the retrieval of spatial memories rather than temporal sequencing.

Process analyses by Brown (1976) support the intuition that backward reconstruction of a route is cognitively more demanding than forward reconstruction. This difference in performance requirements is important, because in some instances forward reconstruction is at such high levels that the effects of age or representational abilities cannot be assessed (Cousins, Siegel, \& Maxwell, 1983). In terms of measurement properties, then, a route reversal task may provide sufficient variability in dependent measures so that effects of independent variables may be revealed. However, children of different ages have shown similar performance in reversing a route through an indoor maze, when all have achieved some criterion for learning the route in a forward direction (Allen, Kirasic, \& Beard, 1989; Hazen, Lockman, \& Pick, 1978). These considerations suggest that the development of place recognition abilities may 
be effectively studied by using a reversal task following a single incidental exposure to a route.

The developmental study of route reversal performance may be particularly informative for process analyses. For example, recent investigations have shown that 6- and 8-year-old children are more likely than 12year-old children to step off route when they are asked to lead the way back along a route they have just walked (Cornell, Heth, \& Broda, 1989; Cornell, Heth, Kneubuhler, \& Seghal, 1994; Cornell, Heth, \& Rowat, 1992). In addition, after mistakenly stepping off route, 6- and 8 -year-old children often fail to get back on route and hence tend to wander more than do 12-year-old children. In general, when a number of measures of route reversal performance were examined, the way finding of 12 year-old children generally did not differ from that of 22-year-old adults (Cornell et al., 1992).

A variety of developments in spatial representation and reasoning may underlie such age-related differences in route reversal performance. By 12 years of age, children seem to be able to estimate distance traveled, orient to locations that are not immediately visible, infer shortcuts, and use procedures to correct navigation errors (Cohen, 1985; Liben, Patterson, \& Newcombe, 1981; Spencer, Blades, \& Morsley, 1989; Stiles-Davis, Kritchevsky, \& Bellugi, 1988). These cognitive abilities would facilitate way finding in unfamiliar environments only if the child had first attended to landmarks and remembered relations between landmarks that indicated the distance and direction of travel. Hence, the ability to recognize landmarks is considered to be a fundamental development underlying way-finding performance (Siegel \& White, 1975).

Although visual recognition memory for isolated objects appears to be robust and shows little change during the course of child development (Brown, 1975; Kail \& Siegel, 1977), there is evidence of age-related improvement in the processing of complex scenes (Azmitia, Merriman, \& Perlmutter, 1987; Hock, Romanski, Galie, \& Williams, 1978; Mandler \& Robinson, 1978; Sophian \& Stigler, 1981). Most pertinent to the present inquiry is a developmental investigation of recognition of landmarks in pictures of the environment (Kirasic, Siegel, \& Allen, 1980). In this investigation, 6-year-old children, when compared with 10 - and 22 -year-old subjects, were found to be inaccurate and slow at identifying photographs of European locations they had studied. Each photograph that they had studied portrayed a central landmark (building, bridge, or fountain) that could be used readily as a cue for way finding. Some recognition tests included pictures of landmarks extracted from the previously studied environmental scenes and isolated on blank backgrounds. The pattern of results indicated that the younger children had less difficulty in distinguishing landmarks as isolated objects than when the landmarks were pictured in their natural surroundings.

The processes necessary for recognition of landmarks in context would seem to be the same as those necessary for place recognition. However, caution is warranted in generalization from investigations of children's recognition of planar materials such as slide depictions of environmental scenes. Pictorial simulations do not capture the interactive feedback that is obtained from exploration, such as the views of the sides of objects revealed by head movements in the natural environment (Gibson, 1979). Empirically, both younger and older children make fewer navigational errors in actual way-finding tasks than they do when they are asked to simulate navigation in a videotape or slide presentation of a route (Cornell \& Hay, 1984). Hence, it is important to establish and analyze age-related performance in place recognition when tests occur in situ.

Signal detection theory provides a flexible method for characterizing the processes of recognition memory under such conditions (Murdock, 1982). Certain assumptions should be explicit to make predictions about recognition performance and navigation. Memory traces for real-world scenes can be ordered along a single strength axis. Confusions between previously experienced and new scenes occur because of overlap in two distributions of strengths. In the route reversal task, one distribution occurs as a result of previous experience with the landmarks or other events encountered during the original walk. The other distribution represents strength traces activated by new scenes experienced in places similar to those actually encountered. These places off route may be contiguous with the original route and hence may share geographical features or reveal landmarks seen from the original route. Decisions about familiarity are determined by the strength of a trace relative to a subjective cutoff; when the trace activated by a realworld scene exceeds the way finder's cutoff, the way finder judges the place to be familiar.

One way to reverse a previously traveled route is to make navigational decisions on the basis of familiarity. If we assume that a requisite task for the way finder is to discriminate between previously experienced and new paths, judgments of familiarity of places on the previously traveled route will be correct and can be labeled as hits, and judgments of familiarity of places off the previously traveled route will be incorrect and can be labeled as false alarms. As in other applications of signal detection analysis, these assumptions allow identification of different sources of recognition performance. Two parameters of particular relevance for our analysis are $d^{\prime}$, the separation between the mean strength of the two distributions, and $\mathbf{c}^{\mathrm{T}}$, a vector describing the criteria used as cutoffs for judging familiarity (usually referred to as $\beta$ ).

The separation of these two parameters could be particularly informative in a developmental analysis of place recognition used in route reversal. For example, consider the findings regarding age-related differences in navigational errors that we reviewed earlier. The differential tendency of younger children to step off route may be due to inadequate selection and utilization of 
landmarks at intersections on route where navigational decisions are required (Allen, Kirasic, Siegel, \& Herman, 1979). Older children and adults may be more adept at selecting landmarks that are informative for discriminating familiar and novel paths. This would be reflected in an age-related increase in $d^{\prime}$. In addition, older children and adults may be capable of restricting response criteria in a way that optimizes the judgment that a place is novel (e.g., decreases the frequency of false positive errors). This would be reflected in an age-related increase in signal detection criterion levels, as reflected in the values that constitute $\mathbf{c}^{\mathrm{T}}$.

The second age-related difference-the tendency of younger children to have more off-route travel-suggests a relation between contiguous environmental cues and the ability to judge places as novel. Consider a way finder who has wandered only a short distance off route, has turned, and is approaching a crossroads that includes the original route. This is an important site, because it presents an immediate opportunity to get back on route. As the intersection comes into view, landmarks can be seen along the off-route paths that perhaps have been seen previously from the perspective of the original route. In addition, previously seen landmarks can be seen next to the route originally traveled, but these are viewed from a different perspective than when they were first encountered. In such an abundance of partially familiar cues, way finders could have difficulty detecting that they were at a place off route and might continue along an incorrect path. However, environmental features at intersections farther from the original route would have less overlap with features that have been seen adjoining the original route, resulting in greater discriminability between old and new places. In other words, $d^{\prime}$ should increase as a function of novel landmarks, which are encountered increasingly as off-route travel continues away from the original route.

Despite a possible increase in discriminability between old and new places, 6- and 8-year-old children wander more distance from correct routes than do 12 year-old children and adults. This distant wandering occurs even though 6-year-old children freely ask for help when they finally do realize that they are off route (Cornell et al., 1989; Cornell et al., 1992). Cornell and his colleagues suggest as one explanation that young children may press forward on an incorrect path because they have different criteria than do older children and adults for judging their surroundings as familiar.

More generally, after traveling off route for some time without seeing a previously seen landmark, way finders may become anxious that they are off route and attempt to alleviate this anxiety by judging a new landmark as familiar. In other words, to the extent that they are more distant from the original route, travelers seeking familiar landmarks may "grasp at straws."

Taken together, these interpretations predict a pattern of parametric differences when place recognition is assessed at intersections off the original route. First, $d^{\prime}$ should increase with age, indicating the development of place recognition accuracy. Second, there should be a positive relation between distance off route and $d^{\prime}$. This relation obtains as more distant environmental features differ from those seen on the original route. Third, the values underlying $\mathrm{c}^{\mathrm{T}}$ should increase with age, reflecting that 8-year-old children have more liberal criteria for recognizing places off route as familiar than do 12-yearold children and adults. Fourth, the values underlying $\mathbf{c}^{\mathrm{T}}$ should decrease with distance off route, reflecting that, as novel environmental features are increasingly encountered, all age groups liberalize criteria for judging places as familiar.

\section{METHOD}

\section{Subjects}

One hundred and eight people completed the recognition tests. There were thirty-six 8 -year-old children $(M=8: 1$; range $=$ 7:6-8:6), thirty-six 12-year-old children $(M=12: 0 ;$ range $=$ $11: 6-12: 6)$, and 36 adults $(M=25: 3$; range $=18: 0-30: 5)$. There were 18 females and 18 males in each of the three age groups.

The children were recruited from a list of children who had participated in research as infants. Their parents were informed by letter of the sponsorship, purposes, and methods of the study and were subsequently contacted by telephone to discuss the project and schedule appointments. The adults responded to an advertisement in a local newspaper offering $\$ 10$ for $1 \mathrm{~h}$ of participation in psychological research. Participants were not included in the study if it was determined that they had previously visited the campus or if they were informed or inferred that their role included navigating the campus. Seven participants were replaced because they were knowledgeable in these ways.

\section{Routes}

The participants were first escorted on an 872-m route (See Figure 1). This original route took approximately $10 \mathrm{~min}$ to walk at an adult's pace and 12 min to walk at a child's pace. The original route traversed the university campus in a semicircular configuration by following established sidewalks, paved paths, and service roads and passing by a variety of landscaping, architecture, and construction.

At the end of the original route, the participants were stopped, turned $180^{\circ}$, and escorted on one of several return routes (see Figure 1). A return route was designed with branches so that participants could be led off the original route for recognition tests in unfamiliar places. The return route took approximately $40-45 \mathrm{~min}$ to complete. Crossroads or Y- or T-shaped intersections of two or more continuing walkways along the return route were identified as sites for recognition tests. The participants were stopped $7 \mathrm{~m}$ from the center of intersections for recognition tests.

During the return, recognition tests occurred at four sites on the original route and six sites on the off-route branches. The sites for the four recognition tests on the route that had been originally walked were the same for every participant. The sites for the six recognition tests off route differed across participants in a counterbalanced design. Three of these tests were distant from the original route and three were close. The three distant recognition tests were given at the termini of off-route branches, as participants approached either the third or the fifth intersection as they walked away from the original route. The test sites as participants approached the third intersections were a mean of $95 \mathrm{~m}$ from the original route and those as they approached the fifth intersections were a mean of $118 \mathrm{~m}$ from the original route. After the participants had turned back from each of the three off-route excursions, 


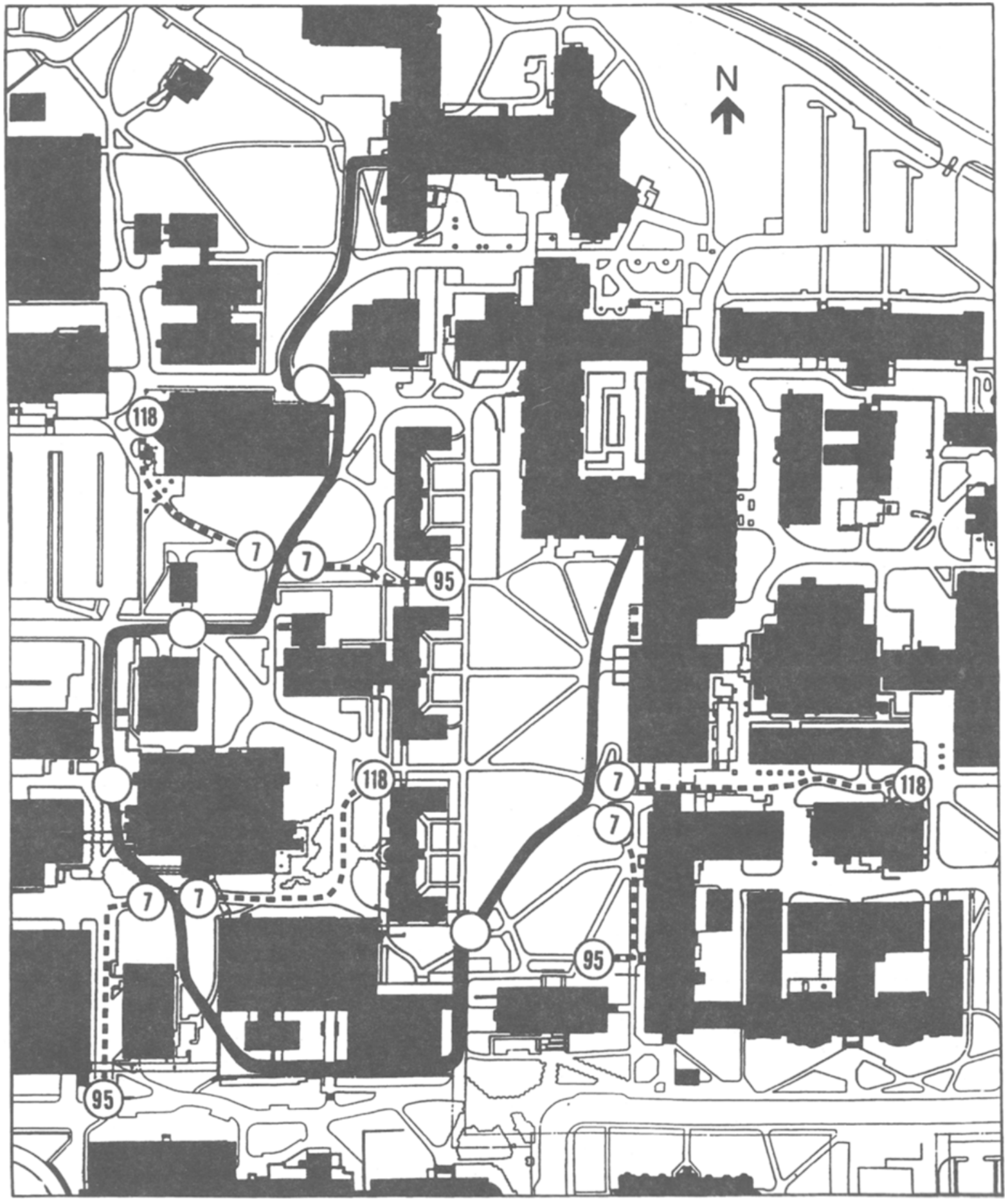

Figure 1. An overhead view of the campus area containing the routes. The solid line shows the original route, and the dashed lines show the optional branches. Sites used for recognition tests are indicated by circles near intersections. The four empty circles mark test sites on route, and the numbered circles mark test sites off route as one of three average distances in meters to the original route.

close recognition tests were given as they approached the intersections of the branches and the original route. Hence, in addition to the three distant recognition tests, each participant was tested for place recognition three times while off route and facing an intersection of the original route from $7 \mathrm{~m}$.

\section{Experimental Conditions}

The independent variables of interest were the age of the participants and the location of the recognition tests. The four tests on the original route were given in view of familiar cues that had been seen previously from travel in the opposite direction. The 
three close tests $7 \mathrm{~m}$ off route also provided views of familiar cues along the original route, but these were available as the original route was approached from a side. The three more distant off-route tests faced away from the original route. The participants may have seen some of the environmental features congruent with these sites as they looked from side to side as they walked the original route. However, the likelihood that these tangential features could be examined in detail and the likelihood of correspondence of these features with cues on the original route decreased with increasing distance from the original route.

There were also several independent variables that were counterbalanced with the variables of interest to provide generality to the results and allow for effective test procedures. Random assignment between subjects ensured that equal numbers of each age and gender received the different test orders and locations.

In pilot studies, some children and adults reported that they inferred they were approaching familiar territory because they had recently turned or had just been asked about a place. Two procedural foils were used to ensure that recognition judgments were not biased by recent test procedures. First, at any of the branches, half of the subjects were led off route to approach either the third or the fifth intersection and recognition tests were given. The other half of the subjects were turned around without being tested for recognition at these distant sites; they were led back to the intersection of the original path, whence they continued on the adjoining branch to approach either the third or the fifth intersection off route for a distant recognition test. The second procedural foil involved recognition tests given as the subjects approached the original route from $7 \mathrm{~m}$ off route. Half the subjects received these tests on the same branch that had been used for giving a distant recognition test; half were tested on the adjoining branch. These foils and the vagaries of turns on the original route were sufficient to eliminate reports that test procedures were used as a cue for judging familiar territory.

Some children participating in pilot studies reported that they became tired of the task when it exceeded $1 \mathrm{~h}$; four distant recognition tests were not possible within this time frame when the offroute excursions included the foils. Hence, three distant recognition tests were given within subjects so that between subjects half of the sample received two tests at about $95 \mathrm{~m}$ and one test at a site about $118 \mathrm{~m}$ off route, and half of the sample received two tests at about $118 \mathrm{~m}$ and one test at about $95 \mathrm{~m}$ off route.

\section{Procedure}

The children and adults were tested individually during the summer months when the campus was relatively free of activity. There were four phases to the testing procedure. First, there was a briefing and familiarization period with the child and parent or with the adult. Next, the participant was led along the original route and informed at the end that there would be tests for recognition of places he/she had viewed incidentally along the way. The participant was then turned around and led along a return route that included predetermined sites for recognition tests. After arrival at the starting point of the original route, participants and parents were debriefed as to the purposes of the research, the reasons for the unexpected test procedures, and a general description of group performance. Scripts for the briefings and test procedures were memorized by the tester, but variability in conversations often required that the tester improvise responses to communicate the gist of the procedures.

Upon arrival at the building housing the psychology department, the tester introduced herself to the family or adult and began light conversation with the participant. The purpose of the conversation was to put the participant at ease and to ascertain what the participant anticipated about the activities to come. The participant was asked, "Do you know what we are going to be doing today?" The parents had been instructed to tell their children that they were going to take a tour of the university with a student; the adults were told that they would be tested for problem-solving strategies at a laboratory located across campus. If it became clear that a participant was aware of the true significance of the walk across campus, typical procedures were followed, but the data were not analyzed and the test was reassigned. Permission to participate was obtained as a conclusion to the briefing.

Original route. The original route began in the northwest, at exterior stairs reached by walking through the psychology wing. Conversation was allowed so that children and adults would feel natural during the walk, but the tester was laconic so as not to distract attention to the surroundings. The participant walked alongside the tester in the center of the path.

Return route. Upon arrival at the northeast end point of the original route, all participants were told: "We'll stop here. We have just taken a walk across campus and now I'm going to ask you some questions because we are interested in what people remember along the way back. Did you think that I might be asking you questions about the walk?" The response to this question was used to determine whether a participant could be considered to have learned about places incidentally.

Next, the participants were told:

Now, do you remember the building where we started? I will lead the way back to those stairs. Sometimes I will walk along the path we have just taken. I will call this the original path. Other times I will go off of the path, as though we are just wandering around-But don't worry, I have a map on this clipboard that tells me the exact way to go. I will stop at certain locations and ask you whether you think we have been there before or not. Do you understand what we are going to do? Okay, let's start.

Recognition tests were posed at 10 intersections predetermined for each subject within the counterbalanced design. Prior to arrival at the selected intersections, the tester would slow to position the participant facing the middle and announce, "Okay, let's stop here. Do you think we are off the original path or on the original path?" Then, the participant was shown a confidence scale (a method depicted in Table 1.1 of Murdock, 1982):

Use the ends of this scale to tell me how sure you are about whether you remember this location as part of the original path. For example, if you are very sure that this is a new location, one that was definitely not a part of the original path, point to the three minus signs. If you are very sure that this is an old location, one that was definitely a part of the original path, point to the three plus signs. If you are not that certain that this is a new location or an old one, point to something in between. For example, these two minus signs mean that you are fairly sure that you are off of the original path and this one minus sign means that you are only a little bit sure that you are off the original path; these two plus signs mean that you are fairly sure that you are on the original path and this one plus sign means that you are only a little bit sure that you are on the original path.

After confidence was indicated, the tester said, "Now, we want to return to our starting point by continuing on the original path. Could you point to the way we should go?" To confirm the direction of the point, one of two questions was then posed. If the participant was on the original route or approaching it from $7 \mathrm{~m}$ off route, the tester asked, "Would going in that direction [pointing as indicated by the participant] be the way to go back to the building where we started?" If the participant was 95 or $118 \mathrm{~m}$ off the original route, the tester asked, "Would going in that direction [pointing as indicated by the participant] be the way to go back to the place where we left the original path?" If the participant said "yes," the tester responded, "Good, let's keep going. I'll lead the way." If the participant said "no," the tester asked the participant to point to the path that would take them to the respective goal. After confirming the direction of the point, the tester then led the participant to the next choice point.

Some off-path excursions ended without a recognition test being posed. For this procedure, the tester stopped prior to designated intersections and announced, "Okay, let's turn here." No ex- 
planation was given for this turn and retracing of steps, but if the participant commented or questioned the procedure, the tester said, "Don't worry, I'm leading the way and I know exactly where we are going."

Throughout the return walk, the tester avoided explicit reinforcement of comments and answered many questions with a question. For example, if a child on one of the branches off of the original route asked, "Are we going the right way?" the tester would respond, "Are we going the right way?-Don't worry, I'm leading the way and I know exactly where we are."

The test procedure was declared to be complete upon sight of the stairs that marked the start of the original route. After walking through the building to the psychology department, all participants were shown the original and return routes on a large map of the campus. Even some of the younger children were able to reconstruct portions of their walk by referring to the map. The participants were thanked and were told that a summary of the results would be mailed upon completion of the study.

\section{RESULTS}

\section{Signal Detection Analysis}

To analyze recognition performance, the logistic version of the signal detection model (Ogilvie \& Creelman, 1968) was employed. This version simplifies the numerical calculation of parameters by using a logistic distribution to approximate the normal curve. Estimates of $d^{\prime}$ and the vector of judgment criteria (c' $\mathbf{c}^{\mathrm{T}}$, comprising the values $c_{1}$ through $c_{5}$ ) were obtained by solving the maximum likelihood equations given by Ogilvie and Creelman. The equations were solved using starting values of $d^{\prime}=1.5$, and values of $\mathbf{c}^{\mathrm{T}}$ were set to $1.0,0.5,0$, -0.5 , and -1.5 . The initial ratio of the variances of the two distributions $(r)$ was set to 1 .

The values for the parameters of the Ogilvie and Creelman (1968) model were then estimated by using a jackknife procedure (Tukey, 1969). This procedure provides estimates of $d^{\prime}$ and $\mathbf{c}^{\mathrm{T}}$ for the different age groups by iteratively calculating the model with 2 subjects from an age group omitted. Different subjects are omitted on different iterations, providing a distribution of estimates. The resulting group estimates are given in Table 1. Estimates of $d^{\prime}$ and $\mathbf{c}^{\mathrm{T}}$ for recognition tests made at different mean distances from the original route were likewise obtained from the jackknife procedure, by

Table 1

Estimated Parameters, With Standard Errors, of the Signal Detection Model for Age Groups

\begin{tabular}{|c|c|c|c|c|c|c|}
\hline \multirow{3}{*}{$\begin{array}{l}\text { Parameters } \\
\text { of the Model }\end{array}$} & \multicolumn{6}{|c|}{ Age (in Years) } \\
\hline & \multicolumn{2}{|c|}{8} & \multicolumn{2}{|c|}{12} & \multicolumn{2}{|c|}{25} \\
\hline & Est. & $S E$ & Est. & $S E$ & Est. & $S E$ \\
\hline$d^{\prime}$ & 1.54 & $0.21_{a}$ & 2.31 & 0.31 & 2.34 & 0.20 \\
\hline$c_{1}$ & 1.68 & $0.27_{\mathrm{a}}$ & 3.04 & 0.54 & 2.40 & 0.29 \\
\hline$c_{2}$ & 1.03 & $0.20_{\mathrm{a}}$ & 1.73 & 0.31 & 1.72 & 0.18 \\
\hline$c_{3}$ & 0.68 & $0.20_{\mathrm{a}}$ & 1.22 & 0.28 & 1.43 & 0.16 \\
\hline$c_{4}$ & 0.26 & $0.15 \mathrm{a}$ & 0.66 & 0.23 & 1.05 & 0.18 \\
\hline$c_{5}$ & -0.72 & $0.18_{\mathrm{a}}$ & -0.27 & $0.25_{b}$ & 0.26 & 0.19 \\
\hline
\end{tabular}

Note $-c_{1}-c_{5}$ are values of $\mathbf{c}^{\top}$, the vector describing the criteria used as cutoffs for judging familiarity. Estimates within a row with different subscripting differ by more than the sum of their standard errors.
Table 2

Estimated Parameters, With Standard Errors, of the Signal Detection Model for Test Distances (in Meters) From the Original Route

\begin{tabular}{|c|c|c|c|c|c|c|}
\hline \multirow{3}{*}{$\begin{array}{l}\text { Parameters } \\
\text { of the Model }\end{array}$} & \multicolumn{6}{|c|}{ Distance } \\
\hline & \multicolumn{2}{|c|}{7} & \multicolumn{2}{|c|}{95} & \multicolumn{2}{|c|}{118} \\
\hline & Est. & $S E$ & Est. & $S E$ & Est. & $S E$ \\
\hline$d^{\prime}$ & 1.64 & $0.19 \mathrm{a}$ & 2.58 & 0.18 & 2.37 & 0.21 \\
\hline$c_{1}$ & 1.82 & $0.25 \mathrm{a}$ & 3.17 & 0.34 & 3.02 & 0.40 \\
\hline$c_{2}$ & 0.94 & $0.17_{\mathrm{a}}$ & 1.98 & 0.25 & 1.88 & 0.24 \\
\hline$c_{3}$ & 0.65 & $0.15_{\mathrm{a}}$ & 1.52 & 0.22 & 1.38 & 0.19 \\
\hline$c_{4}$ & 0.25 & $0.13 \mathrm{a}$ & 1.17 & 0.22 & 0.88 & 0.17 \\
\hline$c_{5}$ & -0.52 & $0.13_{a}$ & 0.37 & $0.18_{b}$ & -0.06 & 0.16 \\
\hline
\end{tabular}

Note- $-c_{1}-c_{5}$ are values of $\mathbf{c}^{\mathrm{T}}$, the vector describing the criteria used as cutoffs for judging familiarity. Estimates within a row with different subscripting differ by more than the sum of their standard errors.

systematically omitting 3 subjects for each iteration. One subject from each age group was omitted for each of these iterations. The resulting group estimates are given in Table 2.

Table 1 indicates that an effect of age on $d^{\prime}$ appears to be located in the difference between the 8-year-olds and the two older groups, which do not appear to differ. The smallest values of $d^{\prime}$ indicate that the youngest travelers were least able to distinguish old from new places. Table 2 indicates the mean distance of the site for the recognition test from the original route also seemed to affect $d^{\prime}$ differentially. When way finders were 95 and $118 \mathrm{~m}$ from the original route, their ability to distinguish old and new places was greater than when they were approaching the intersection of the original route from $7 \mathrm{~m}$ off route.

It is difficult to test these apparent differences according to standard statistical techniques, because each subject does not provide an independent measure of the value of a parameter. That is, $d^{\prime}$ could not be estimated for individual subjects because the length of the route limited the number of recognition tests that could be given to way finders.

However, we can provide a general index of group differences by using the Tukey jackknife procedure. The jackknife procedure generates estimates of the standard error of statistics such as $d^{\prime}$ and $\mathbf{c}^{\mathrm{T}}$. If we assume normal distributions of these statistics, a good heuristic to detect group differences is to look for distributions for which the standard errors do not overlap. This heuristic indicates that the description of group differences apparent in Tables 1 and 2 is correct.

The vector $\mathbf{c}^{\mathrm{T}}$ is a measure of the criteria used by the way finders to denote their confidence of a recognition judgment. Recognition confidence under the Ogilvie and Creelman (1968) model is determined by the strength of a memory trace along a single dimension. There are five values of $\mathbf{c}^{\mathrm{T}}$ that delimit regions along the strength dimension corresponding to the six different confidence categories of very sure, fairly sure, etc. For example, the value of $c_{1}$ marks the lower boundary of the 
very sure-familiar judgment. Any trace higher in magnitude will produce this judgment.

The group differences in the values constituting $\mathbf{c}^{\mathrm{T}}$ are similar to those for $d^{\prime}$. Table 1 indicates that the first value $\left(c_{1}\right)$ used to estimate the criteria for recognition of the 8-year-olds differs from that of the 12- and 25-yearolds. The lower value of $c_{1}$ indicates that the youngest children had a lower threshold for indicating that they were very sure of being on the original route. Other values constituting $\mathbf{c}^{\mathrm{T}}$ preserve the original difference, suggesting that the scale for making recognition judgments is shifted for the youngest subjects. They require less evidence to judge that they are on the original route; when off route, they are correspondingly less likely to judge the route as novel.

Table 2 indicates a parallel pattern in $\mathbf{c}^{\mathrm{T}}$ when subjects are tested at different test sites off the original route. When estimated at test sites approaching the intersection of the original route from $7 \mathrm{~m}$ off route, $c_{1}$ differs from that for intersections 95 or $118 \mathrm{~m}$ off route. Other values preserve this initial difference. It appears that way finders close to and facing the original route require less evidence when they judge their location as being on path. The context of approaching the original path seems to have special consequences.

\section{Recognition Errors}

The differences in criteria for place recognition can be clarified by examining the patterns of errors that occurred. For example, a false positive error occurs when a way finder is not on the original route but mistakenly judges a place off route as familiar. An analysis of false positive errors can indicate how the criteria for place recog-

Table 3

Mean Per Cent Errors and Number of Familiar Judgments Made On and Off the Original Route

\begin{tabular}{rlc}
\hline Age in Years & Per Cent of Errors & Familiar Judgment \\
\hline \multicolumn{3}{c}{ On route } \\
8 & 27 & 105 \\
12 & 22 & 113 \\
25 & 26 & 106 \\
& Off $7 \mathrm{~m}$ & \\
8 & 47 & 51 \\
12 & 27 & 29 \\
25 & 20 & 22 \\
& Off $95 \mathrm{~m}$ & \\
8 & 21 & 11 \\
12 & 19 & 10 \\
25 & 14 & 8 \\
& Off $118 \mathrm{~m}$ & \\
8 & 15 & 9 \\
12 & 11 & 10 \\
25 & 17 & \\
\hline
\end{tabular}

Note-Each age group made the same number of judgments at each location. There were 144 judgments on the original route, 108 judgments averaging $7 \mathrm{~m}$ away, 54 judgments averaging $95 \mathrm{~m}$ away, and 54 judgments averaging $118 \mathrm{~m}$ away. *Errors on route were false negatives; errors off route were false positives. nition change as individual way finders are at different sites off the original route.

Because different subjects received different numbers of tests at different distances off route, the dependent measure for analysis is the percentage of judgments that were false positive errors. The analysis was a repeated measures multivariate analysis of variance (MANOVA). The between-subjects factor was age $(8,12$, or 25 years), and the within-subjects factor was the average distance that the recognition test was off the original route $(7,95$, or $118 \mathrm{~m}$ ).

There was a significant main effect of distance $\left[F(2,210)=9.884, M S_{\mathrm{e}}=0.887, p<.001\right]$. There was also an interaction of age and distance $[F(2,105)=$ $\left.4.289, M S_{\mathrm{e}}=0.084, p<.02\right]$. These effects can be interpreted by examining Table 3 , which lists the per cent errors calculated for each individual and then averaged across. It appears that false positive errors generally decreased when participants were distant and facing away from the original path. This evidence is counter to the notion that criteria for recognition are liberalized in new territory. Especially high percentages of false positive judgments were obtained when the 8-year-old children were tested at the locations where the original path could be seen from $7 \mathrm{~m}$.

Table 3 also lists false negative errors, which occur when a way finder is on the original route and mistakenly judges a place as new. The percentage of judgments that were false negative errors may indicate the extent to which places passed during the original walk looked different from the perspectives during the return. There were no reliable differences between the age groups $\left[F(2,105)=0.560, M S_{\mathrm{e}}=0.059\right]$.

\section{Directional Judgments}

After being tested for place recognition near intersections, way finders were asked to point to the path that would allow return to the starting point by continuing on the original route. These directional judgments permit an analysis of the relation between recognition accuracy and navigation. Including that the way finders must often point in the direction opposite to the one they were facing, the modal number of choices at intersections was four. If the way finder was being tested on the original route or approaching the original route from $7 \mathrm{~m}$, a point to the path that reversed the original walk was defined as a correct judgment. If the way finder was being tested off the original route from 95 or $118 \mathrm{~m}$, a point backward to the path that led back to where he/she had left the original route was defined as a correct judgment.

Because different subjects received different numbers of tests at different distances off route, the dependent measure for analysis is the percentage of correct directional judgments. The analysis was a MANOVA including repeated measures. The between-subjects factor was age $(8,12$, or 25 years) and the within-subjects factor was the average distance that the recognition test was off the original route $(0,7,95$, or $118 \mathrm{~m})$. 
Table 4

Mean Per Cent of Recognition Judgments Associated With Correct Directional Judgments

\begin{tabular}{|c|c|c|c|c|c|c|c|c|}
\hline \multirow{3}{*}{$\begin{array}{c}\text { Recognition } \\
\text { Judgment }\end{array}$} & \multicolumn{8}{|c|}{ Age in Years } \\
\hline & \multicolumn{2}{|c|}{8} & \multicolumn{2}{|c|}{12} & \multicolumn{2}{|c|}{25} & \multicolumn{2}{|c|}{ Age Effect } \\
\hline & $\%$ & $n$ & $\%$ & $n$ & $\%$ & $n$ & $d f$ & $F$ \\
\hline \multicolumn{9}{|c|}{ On Route } \\
\hline True positive & 71 & 35 & 75 & 35 & 76 & 35 & 2,102 & 0.290 \\
\hline False negative & 36 & 25 & 43 & 21 & 63 & 26 & 2,69 & 2.330 \\
\hline \multicolumn{9}{|c|}{ Off $7 \mathrm{~m}$} \\
\hline True negative & 45 & 28 & 80 & 33 & 76 & 34 & 2,92 & $9.211^{*}$ \\
\hline False positive & 50 & 28 & 69 & 18 & 69 & 14 & 2,57 & 1.482 \\
\hline \multicolumn{9}{|c|}{ Off $95 \mathrm{~m}$} \\
\hline True negative & 53 & 32 & 90 & 31 & 79 & 33 & 2,93 & $8.092 *$ \\
\hline False positive & 0 & 11 & 0 & 11 & 14 & 7 & $\mathrm{n}$. & v. \\
\hline \multicolumn{9}{|c|}{ Off $118 \mathrm{~m}$} \\
\hline True negative & 36 & 33 & 65 & 34 & 68 & 33 & 2,97 & $4.817^{*}$ \\
\hline False positive & 13 & 8 & 0 & 6 & 6 & 9 & \multicolumn{2}{|c|}{ n.v. } \\
\hline
\end{tabular}

There were significant main effects of age $[F(2,105)=$ 13.704, $\left.M S_{\mathrm{e}}=0.185\right]$ and distance $[F(3,315)=6.103$, $\left.M S_{\mathrm{e}}=0.113, p \mathrm{~s}<.001\right]$. No other effects were reliable. Tukey HSD tests $(p<.05)$ indicated that the percentage of correct directional judgments by 8 -year-olds $(M=$ $45 \%$ ) differed from those of 12 - and 25 -year-olds, which did not differ from each other $(M \mathrm{~s}=68 \%$ and $69 \%$, respectively). A linear trend characterized the group differences in pointing accuracy at different distances $\left[F(1,105)=14.083, M S_{\mathrm{e}}=0.130, p<.0001\right]$. The mean of correct directional judgments on route was $67 \%$, and the means for judgments 7,95 , and $118 \mathrm{~m}$ off route were $66 \%, 60 \%$, and $50 \%$ correct, respectively.

\section{Concordance of Place Recognition and Directional Judgments}

Here we estimated the relation between place recognition and navigation choices. We distinguished the two types of recognition judgments possible at intersections on and off the original route as correct or erroneous. We then calculated the percentage of each type that was followed by the way finder pointing the correct direction to proceed. These percentages are listed in Table 4 with results of analyses of variance (ANOVAs) testing the effects of age.

Separate ANOVAs were required for the different locations of the test intersections because several subjects did not contribute certain recognition judgments at all distances. However, the results of the separate ANOVAs listed in Table 4 were consistent; age effects were present in all true negatives. Tukey HSD tests $(p<.05)$ indicated that 8-year-old children had lower percentages of correct directional judgments following a correct recognition that they were off the path; 12- and 25-yearold way finders did not differ with regard to their greater percentages of correct directional judgments following recognition that they were off the path.

\section{DISCUSSION}

The most revealing portions of the route reversal task occurred when children and adults were tested for place recognition after being led off of the original route. Table 3 indicates that, when on route, members of all three age groups show comparable intermediate levels of recognition performance. When off route, however, an interaction of age and test location was observed. The youngest children made considerably more false positive errors, especially at the locations that afforded views of the original route.

Analysis of recognition performance using a model of signal detection (Ogilvie \& Creelman, 1968) suggests a developmental account of this pattern. The model indicated that $d^{\prime}$-the parameter characterizing recognition accuracy-was lower for the 8-year-old children than for the 12-year-old children and 25-year-old adults. The values we obtained are consistent with estimates of $d^{\prime}$ in a pertinent scene recognition task (Doherty \& Pellegrino, 1985). In this task, children ranging in age from 7 to 15 years were asked to identify familiar views in slides taken from routes in their own and distractor neighborhoods. Because the younger children were subject to more restrictions in their range of play and travel, the researchers acknowledged a confounding of children's age and extent of experience in the neighborhood. In the present study, the environment was new for all participants, so age-related increases in recognition accuracy can be attributed to the development of recognition processes.

The model further indicated that the superior recognition of the older way finders is not apparent on route because of a compensating development in the use of environmental information to decide that a location is familiar. Analysis of the criteria vector $\mathbf{c}^{\mathrm{T}}$ indicated that the 8-year-old children had lower thresholds for confidence that they were on the original route, whereas the older groups were more conservative in judging locations as familiar. As suggested in our introduction, such differences in criteria may underlie the findings that 6 and 8-year-olds wander greater distances from a familiar route; younger children may be less apt to judge that they are in new territory.

The evidence that, when subjects are tested in situ, there are developmental changes in place recognition is compatible with the results showing age-related improvement in the recognition of complex scenes (Kirasic, Siegel, \& Allen, 1980). It is not only that the complex and encompassing environmental context may add difficulty to judgments of the familiarity of places; the requirements of navigation likely affect the processing of memories as well. For example, recognition accuracy is extremely high when young children are shown pictures of familiar environments such as their own school grounds (Cousins et al., 1983). In contrast, we have found that recognition 
accuracy is intermediate in the context of returning along a novel route following incidental learning.

Our results suggest one reason for decreases in recognition accuracy: Route reversal requires recognition of environmental cues that have previously been seen from a reverse perspective. The analyses using the signal detection model indicated that $d^{\prime}$ was lowest at tests approaching the original route from $7 \mathrm{~m}$ off route. The analysis of false positive errors localized age differences at these test sites as well. These test sites are informative because the location off route affords views of onroute cues, but from a perspective alongside. The location $7 \mathrm{~m}$ off route also affords reverse views of cues that were seen to be off route from the perspective of the original route. The pattern of results indicates that way finders have difficulty in discriminating such incidentally viewed landmarks in their different orientations. For young children in particular, the presence of a number of previously seen landmarks may be sufficient to indicate that a place is familiar.

However, way finders who were taken off route for 95 or $118 \mathrm{~m}$ and were facing environmental cues they may have seen from the original route were not more accepting of equivocal evidence of familiarity. Analysis of the criteria $\mathbf{c}^{\mathrm{T}}$ at different test sites indicated that subjects had relatively high thresholds for judging these distant locations as familiar. This result is contrary to the prediction that wandering far off route will cause way finders to liberalize their criteria for judging a place as familiar. We had expected that the decreasing frequency of familiar cues would bias the off-path traveler toward making false positive judgments. Instead, it seems that the accumulated exposure of novel cues during an excursion off route biases a way finder toward making negative judgments. Conversely, way finders who increasingly encounter familiar cues while reversing an off-route excursion may change their criteria so as to be more likely to accept locations close to the original route as familiar.

If recognition accuracy depends on such factors, it follows that one should consider the accuracy of navigation decisions at intersections that were correctly recognized. In general, accuracy of directional choices increased as way finders were closer to the original route. When asked to point to the path for returning to the building at the start, way finders of all ages who correctly recognized that they were on route showed good directional judgments (see Table 4). However, when way finders who correctly recognized that they were off route were asked the direction for returning, this high level of pointing accuracy was maintained only by the two oldest age groups. At all distances off route, the 8-year-olds who knew they were not on the original route were less likely to know the way to proceed than the 12- and 25year-olds. The dissociation between recognition accuracy and pointing accuracy indicates age-related differences in the use of recognition judgments for navigation. Below, we develop a model that is extended to account for such age-related differences.

\section{A Model of Way Finding by Place Recognition}

There is a simple way in which a way finder can repeat a route from beginning to end point: the way finder need only approach those places along the way that are most familiar. The algorithm can be illustrated when travel has begun in the correct direction and a choice of paths is required at the first intersection. A way finder can judge the relative familiarity of choices by looking down the alternative paths as the intersection reveals them. For one or more of the alternatives, landmarks along the path should be unfamiliar because previous travel has not provided close or extended experience with those landmarks. Landmarks along one of the alternative paths should be familiar because they have been seen both in the background and in the foreground as their place was approached. In addition, because the visual field of the traveler is usually oriented toward the direction of movement, landmarks in the front receive more exposure than landmarks in the periphery or rear during the original experience along the route. These observations indicate that the highest familiarity would occur when the original path is viewed in the original direction of travel. Hence, whenever a decision is required for directing travel from the beginning to the end point of a route, the correct choice is to go toward the route features that yield the strongest memories.

Likewise, there is a simple algorithm that can be used for route reversal-repeating a route from the end point to the beginning. The algorithm requires only that the way finder judge the familiarity of places along the route and approach those that are of intermediate familiarity. The algorithm can be illustrated when a way finder has completed the route from the beginning to the end point and has turned around and initiated route reversal in the correct direction. Next, let us assume that an intersection encountered midway during the route reversal is a crossroads where a turn is required. The way finder can judge the relative familiarity of choices by looking down the alternative paths as the intersection reveals them. For two of the alternatives, landmarks down along the paths should be unfamiliar because previous travel has not provided close or extended experience with those landmarks. Landmarks to the rear should be highly familiar because they have been seen previously during travel from the beginning to the end point and most recently during travel initiated at the end point. The correct alternative for route reversal is of intermediate familiarity; as the path is examined, landmarks will have been seen before, but their topological and perspective relations will be shifted systematically from their appearance when they were first experienced while the route was traveled. For example, during travel from the beginning of the route to its end point, a fallen tree was on the right of the path, and the stump of the fallen tree was in the foreground. During travel from the end point to the beginning, the fallen tree was on the left of the path, and the stump of the fallen tree was in the background. 
Note that this algorithm--reverse a route by approaching places of intermediate familiarity-requires only that place recognition be accurate enough for one to make ordinal rankings of familiarity. Directional choices along the return do not require a map-like representation or a list of landmarks and associated actions, or even expectancies of landmarks. Hence, the algorithm could be especially useful when a traveler has not completely acquired or structured spatial or serial information about a route. Young children who lack the cognitive abilities required for organized spatial representations may rely on judgments of the familiarity of places for navigation. However, the poorer recognition accuracy of younger children could result in confusion between ordinal levels of familiarity and produce irregularities in directional choices.

\section{Application and Extension of the Model}

The results obtained in the present study indicate how the model could be applied to a general description of the interactions of recognition and navigation processes. Consider, first, the performance of an individual returning on route and encountering a familiar intersection. In our campus environment, way finders recognized these choice points about $75 \%$ of the time. Furthermore, after correctly recognizing that they were on route, they were able to point to the correct direction in which to proceed with an accuracy of about $74 \%$. There were no age effects in these tendencies, suggesting that developmental differences in way finding are not attributable to performance in the presence of familiar cues.

However, way finders on route were not infallible at either recognizing familiar intersections or at pointing to the correct direction to take. Their intermittent errors indicate that if they had been unguided they would have wandered off route occasionally. This conclusion suggests that, even in the case of route reversal, a complete description of way finding would need to address processes of recognition and navigation in new territory.

An important component of successful route reversal is sensitivity to environmental cues that indicate that a path at an intersection is new. Our data show two developments in this regard. The first is development of recognition accuracy indicated by the age effect in $d^{\prime}$. Most pertinent is the ability to detect novelty indicated by true negative judgments made when one is off path. Older children and adults are better at these judgments when facing a familiar intersection from $7 \mathrm{~m}$, but the 8-yearolds show comparable abilities only when tested facing away from the original route at the farther distances. The second development is a difference between the 8-yearolds and the other age groups in criteria for recognizing a place. Our application of signal detection analysis indicates that the youngest children are willing to accept more novel cues while judging that a place is familiar.

Both of these results suggest that younger children realize that they are off route later than do older children and adults. Such a delay may have implications for the ability to navigate off route. For example, a common strategy in the face of novel cues along a path is to attend selectively to a place where things begin to look different. This registration provides an anchor for subsequent travel and a place to return to. In anticipation of retracing, a way finder could keep track of turns from the anchor point (Loomis, Klatzky, \& Golledge, 1993; Trowbridge, 1913). In other words, the detection of novel cues could trigger a shift from navigation by approaching familiarity with more strategic forms of navigation. If we assume that some 8-year-old children know a prospective strategy such as keeping track of movements from a place, our estimates of their recognition performance indicate that they may not produce it as early in off-route travel as do the 12- and 25-year-olds.

If we assume that some 8-year-old children do not know a prospective strategy for navigation off route, the model of way finding by approaching familiar cues may account for their performance. These children should be at a loss in the absence of familiar cues, and indeed our results indicated that the accuracy of directional judgments made by the 8-year-old children was relatively low even when they correctly recognized that they were off route.

Moreover, the model explains how children who base their navigation decisions on the judged familiarity of places would have difficulty if they did decide to retrace their steps when off route. The difficulty is that the environmental cues seen during off-route travel are partially familiar as they are encountered from the perspective of the retracing. These partially familiar cues must be discriminated from those along the original route, which may also be seen from a new perspective. In other words, while retracing, young way finders who have wandered farther off route must make more judgments involving subtle degrees of familiarity. Their lesser recognition accuracy would likely affect their ability to execute an efficient retrace strategy.

We are suggesting that, with development, processes of place recognition not only provide a tendency to approach a path, but are instrumental in initiating and directing more strategic forms of navigation. Of course, the interactions of place recognition and navigation strategies can be studied directly. Of particular interest would be the effect of manipulations of landmarks at intersections where actions are required. Ethologists have found that such manipulations can reveal the navigational systems of a variety of species (Gallistel, 1990).

\section{REFERENCES}

Allen, G. L., Kirasic, K. C., \& Beard, R. L. (1989). Children's expressions of spatial knowledge. Journal of Experimental Child Psychology, 48, 114-130.

Allen, G. L., Kirasic, K. C., Siegel, A. W., \& Herman, J. F. (1979). Developmental issues in cognitive mapping: The selection and utilization of environmental landmarks. Child Development, 50, 10621070.

Azmitia, M., Merriman, W. E., \& Perlmutter, M. (1987). A lifespan study of the interaction of selectivity and knowledge in memory. Child Development, 58, 276-281.

Brown, A. L. (1975). The development of memory: Knowing, knowing about knowing, and knowing how to know. In H. W. Reese (Ed.), 
Advances in child development and behavior (Vol. 10, pp. 103-152). New York: Academic Press.

BRown, A. L. (1976). The construction of temporal succession by preoperational children. In A. Pick (Ed.), Minnesota Symposium on Child Psychology (Vol. 10, pp. 28-83). Minneapolis: University of Minnesota Press.

COHEN, R. (Ed.) (1985). The development of spatial cognition. Hillsdale, NJ: Erlbaum.

CornelL, E. H., \& Hay, D. H. (1984). Children's acquisition of a route via different media. Environment \& Behavior, 16, 627-641.

Cornell, E. H., Heth, C. D., \& Broda, L. S. (1989). Children's wayfinding: Response to instructions to use environmental landmarks. Developmental Psychology, 25, 755-764.

Cornell, E. H., Heth, C. D., KNeubuhler, Y., \& Sehgal, S. (1994). Serial position effects in children's route reversal errors: Implications for search operations. Unpublished manuscript, University of Alberta, Center for Research in Child Development, Edmonton, Canada.

Cornell, E. H., Heth, C. D., \& Rowat, W. L. (1992). Wayfinding by children and adults: Response to instructions to use look-back and retrace strategies. Developmental Psychology, 28, 328-336.

Cousins, J. H., Siegel, A. W., \& MAXWELl, S. E. (1983). Way finding and cognitive mapping in large-scale environments: A test of a developmental model. Journal of Experimental Child Psychology, $35,1-20$.

DoherTy, S., \& Pellegrino, J. W. (1985). Developmental changes in neighborhood scene recognition. Children's Environments Quarterly, 2, 38-43.

GALLISTEL, C. R. (1990). The organization of learning. Cambridge, MA: MIT Press.

GiBSON, J. J. (1979). The ecological approach to visual perception. Boston: Houghton Mifflin.

Hazen, N. L., Lockman, J. J., \& Pick, H. L. (1978). The development of children's representations of large-scale environments. Child Development, 49, 623-636.

Hock, H. S., Romanski, L., Galie, A., \& Williams, G. S. (1978). Real-world schemata and scene recognition in adults and children. Memory \& Cognition, 6, 423-431.

KaIL, R. V., \& SIEgEL, A. W. (1977). The development of mnemonic encoding in children: From perception to abstraction. In R. V. Kail $\&$ J. W. Hagen (Eds.), Perspectives on the development of memory and cognition (pp. 61-88). Hillsdale, NJ: Erlbaum.

Kirasic, K. C., Siegel, A. W., \& Allen, G. L. (1980). Developmen- tal changes in recognition-in-context memory. Child Development, 51, 302-305.

Liben, L. S., Patterson, A. H., \& Newcombe, N. (Eds.) (1981). Spatial representation and behavior across the life span: Theory and application. New York: Academic Press.

LoOMIs, J. M., Klatzky, R. L., \& Golledge, R. G. (1993, August). Path integration: A neglected form of human navigation. In $\mathrm{H}$. Heft (Chair), Navigation and environmental cognition: Ecological considerations (Symposium conducted at the VIIth International Conference on Event Perception and Action, Vancouver, BC, p. 81.)

MANDleR, J. M., \& Robinson, C. A. (1978). Developmental changes in picture recognition. Journal of Experimental Child Psychology, 26, 122-136.

MurDock, B. B. (1982). Recognition memory. In C. R. Puff (Ed.), Handbook of research methods in human memory and cognition (pp. 1-26). New York: Academic Press.

Ogilvie, J. C., \& Creelman, C. D. (1968). Maximum likelihood estimations of receiver operating characteristic curve parameters. Journal of Mathematical Psychology, 5, 377-391.

PIAGET, J. (1969). The child's conception of time. London: Routledge \& Kegan Paul.

SIEGEL, A. W., \& WhITE, S. H. (1975). The development of spatial representations of large-scale environments. In H. Reese (Ed.), $A d$ vances in child development and behavior (Vol. 10, pp. 9-55). New York: Academic Press.

Sophian, C., \& STIGLER, J. W. (1981). Does recognition memory improve with age? Journal of Experimental Child Psychology, 32, 343-353.

SPencer, C., Blades, M., \& Morsley, K. (1989). The child in the physical environment: The development of spatial knowledge and cognition. New York: Wiley.

Stiles-Davis, J., Kritchevsky, M., \& Bellugi, U. (Eds.) (1988). Spatial cognition: Brain bases and development. Hillsdale, NJ: Erlbaum.

SYROTUCK, W. G. (1979). Analysis of lost person behavior: An aid to search planning. Westmoreland, NY: Arner.

TrowBridgE, C. C. (1913). Fundamental methods of orientation and imaginary maps. Science, 38, 888-897.

TUKEY, J. W. (1969). Analyzing data: Sanctification or detective work? American Psychologist, 24, 83-91.

(Manuscript received September 22, 1993; revision accepted for publication February 7, 1994.) 